\title{
1 Optogenetic Manipulation of Cell Migration with High Spatiotemporal Resolution Using
}

\section{Lattice Lightsheet Microscopy}

3 Wei-Chun Tang ${ }^{1}$, Yen-Ting Liu ${ }^{1}$, Cheng-Han Yeh ${ }^{1}$, Yi-Ling Lin ${ }^{2,3}$, Yu-Chun Lin ${ }^{4,5}$, Tsui-Ling

$4 \mathrm{Hsu}^{6}$, Liang Gao ${ }^{7}$, Shu-Wei Chang ${ }^{1}$, Peilin $\mathrm{Chen}^{1,8, *}$, and Bi-Chang Chen ${ }^{1, *}$

5

$6 \quad{ }^{1}$ Research Center for Applied Sciences, Academia Sinica, Taipei 11529, Taiwan

$7 \quad 2$ Institute of Biomedical Sciences, Academia Sinica, Taipei 11529, Taiwan

$8 \quad{ }^{3}$ Biomedical Translation Research Center, Academia Sinica, Taipei 11529, Taiwan

$9{ }^{4}$ Institute of Molecular Medicine, National Tsing Hua University, Hsinchu 30013, Taiwan

$10{ }^{5}$ Department of Medical Science, National Tsing Hua University, Hsinchu 30013, Taiwan

$11{ }^{6}$ Genomics Research Center, Academia Sinica, Taipei 11529, Taiwan

$12{ }^{7}$ Key Laboratory of Structural Biology of Zhejiang Province, School of Life Sciences,

13 Westlake University, Hangzhou, Zhejiang 310024, China

$14{ }^{8}$ Institute of Physics, Academia Sinica, Taipei 11529, Taiwan

$15 *$ Authors to whom correspondence should be addressed: P.C. (email:

16 peilin@gate.sinica.edu.tw) or B.-C.C. (email: chenb10@gate.sinica.edu.tw) 


\section{Abstract}

19 Lattice lightsheet microscopy (LLSM) is modified with the aim of manipulating cellular

20 behavior with subcellular resolution through three-dimensional (3D) optogenetic activation. In

21 this study, we report a straightforward implementation of the activation source in LLSM in

22 which the stimulating light can be generated by changing the spatial light modulator (SLM)

23 patterns and the annual masks. As a result, a Bessel beam as a stimulation source is integrated

24 into the LLSM without changing the optical configuration, achieving better spatiotemporal

25 control of photoactivation. We show that the energy power required for optogenetic reactions

26 is lower than $1 \mathrm{nW}$ (or $24 \mathrm{~mW} / \mathrm{cm}^{2}$ ), and membrane ruffling can be activated at different

27 locations within a cell with subcellular resolution. We also demonstrate guided cell migration

28 using optogenetic stimulation for up to $6 \mathrm{~h}$, where 463 imaging volumes are collected, without

29 noticeable damage to cells. 


\section{Introduction}

32 Spatiotemporal manipulation and recording of biological processes, such as cell migration or

33 membrane dynamics with the subcellular resolution, is crucial in understanding these

34 fundamental cellular processes ${ }^{1,2,3}$. However, it is technically challenging for cell manipulation

35 with a precise spatiotemporal resolution, especially in three dimensions $(3 \mathrm{D})^{4,5}$. A common

36 approach for subcellular manipulation can be achieved by optogenetic tools on a conventional

37 microscope such as wide-field or confocal microscope $e^{6,7,8}$. Recording the three-dimensional

38 rapid cellular response at high spatiotemporal resolution can be problematic, where a high

39 numerical aperture (NA) objective is often used to create a tightly focused spot for better

40 confinement of the photoactivation beam. When such a photoactivation beam propagates in the

41 cell, it activates molecules not only in the focal spot but also along the beam path where the

42 out-of-focus activation may not be imaged when a pinhole is used to reject the out of focus

43 signal as in the case of confocal microscopy (the beam paths of different excitation and

44 detection schemes can be found in Figure $\mathrm{S} 1)^{9}$. As a result of tight focusing, an uneven photo-

45 excitation along the beam propagation is created. Therefore, it is desirable to develop a wide-

46 field technique with good sectioning capability to record the optogenetic response. A notable

47 implementation in this regard is the development of lattice light-sheet microscopy (LLSM),

48 which is constructed by an array of Bessel beams, where destructive interference of the

49 coherent Bessel beams minimizes the contribution from sidelobes within the detection zone ${ }^{10}$. 
50 LLSM provides several advantages for high-resolution volumetric imaging of living cells,

51 including reduced phototoxicity in which the excitation energy is distributed over an array of

52 the illumination beams, an optimal balance between the thickness and length of the lightsheet,

53 enabling high spatial resolution imaging with a good optical sectioning capability, and

54 improved signal-to-noise ratio in which the out-of-focus noise is suppressed by the coherent

55 modulation of lattice beams ${ }^{11}, 12,13$. In addition, the lattice patterns also allow easy

56 implementation of super-resolution microscopy using structured illumination ${ }^{14}$.

57 With the help of LLSM, several biological processes, including the dynamics of the

58 immunological synapses, transcription factors, and lymphocyte migration, have been

59 investigated in great detail ${ }^{15,16,17}$. With an additional optical path for illumination, it has been

60 demonstrtaed that photoablation/FRAP/photoactivation experiments ${ }^{18,19,20}$ can be conducted

61 on LLSM. However, it remains challenging to perform experiments requiring optical

62 stimulation, such as subcellular optogenetic applications, in LLSM. In this situation, the lattice

63 beams illuminate the samples at a tilted angle, which results in poor spatial activation of the

64 signaling molecules within a cell, especially along the lightsheet propagation direction. LLSM

65 could be potentially useful in living cells to conduct long-term optogenetic experiments with a

66 very high spatiotemporal resolution at very low phototoxicity if the light stimulation can be

67 integrated into LLSM. In addition, the 3D nature of LLSM may allow local activation of

68 biological processes in a specific subcellular compartment or organelle. In this study, we report 
69 a novel LLSM design for optogenetic experiments. The optical configuration of our system is

70 the same as the LLSM reported previously. In our system, the LLSM is used to monitor cellular

71 behavior, whereas optogenetic molecules are activated by a Bessel beam, whose 3D energy

72 distribution can be precisely controlled by the phase profiles on the SLM. Optically-induced

73 clustering and membrane ruffling, in addition to guided cell migration, can be achieved in our

74 system.

75 To demonstrate the performance of our technique, we conducted several cell-migration-related

76 optogenetic experiments in our system. The traditional approach to inducing cell migration is

77 to create concentration gradients of the growth factors ${ }^{21}$ through diffusion mimicking the native

78 cell migration environment. However, the diffusion gradient is difficult to maintain for precise

79 cell manipulation at the single-cell level. The newly developed optogenetic tools enable cell

80 manipulation through light, where cells are modified genetically to respond to a specific

81 wavelength of light ${ }^{22,23}$. The most common genetic modifications for optogenetic experiments

82 include optically induced heterodimerization (cryptochrome 2-calcium and integrin-binding N-

83 terminal domain [CRY2-CIBN] $)^{24}$ and optically induced clustering (CRY2olig) ${ }^{25}$. In this

84 experiment, we adapted both systems to control cellular behavior. To achieve 3D cellular

85 manipulation in LLSM, we demonstrated that our approach offers three advantages over

86 original LLSM, including 1) controllable activation in 3D, 2) subcellular optogenetic activation

87 and 3) long-term cell manipulation. 


\section{Results}

90 Bessel beam activation in lattice lightsheet microscopy for optogenetics

91 To conduct an optogenetic experiment in LLSM, our system consists of a large parallel array

92 of coherently interfering Bessel beams through the SLM in which individual Bessel beams with

93 selected wavelengths can be used as activation sources by simply changing the patterns on the

$94 \mathrm{SLM}^{26}$ located at the plane conjugated to the imaging plane (Figure 1a and $1 \mathrm{~b}$ ). With a different

95 pattern on the SLM, the lattice beams at the imaging plane can be switched to a stimulating

96 Bessel beam whose $\mathrm{x}$, $\mathrm{y}$ positions can be controlled by different modulations in the SLM

97 patterns, as shown in Figure $1 \mathrm{~b}$. In addition, the lattice beams can be dithered to create a

uniform excitation sheet to obtain 3D images of cells with the sample scanning mode, as shown

in Figure 1c. To demonstrate the advantages of using Bessel beam excitation for optogenetic

101 CRY2-CIBN. In the optically-induced clustering probe, we fused CRY2olig with a mRuby3.

102 As the CRY2 molecules oligomerize upon blue light illumination, the mRuby3 fluorescence

103 proteins move closer together, forming a bright emitting spot (Figure 1d). In this experiment,

104 we tested several activation powers ranging from 0.5 to $4 \mathrm{nW}$ in which the Bessel beam was

105 formed using maximum and minimum numerical apertures (NAs) of 0.64 and 0.56 , 
107 along the beam propagation direction are illustrated in Figure S2 of the Supplementary

108 Information. The time-lapse maximum intensity projection (MIP) images of the CRY2olig-

109 mRuby3 transfected human bone osteosarcoma epithelial cell line(U2OS) with various

110 stimulation energies are shown in Figure 1e. At each time point, a series of images were

111 collected in which the cell was first excited by a shorter wavelength laser for activation and

112 imaged by LLSM with a $10 \mathrm{~ms}$ exposure time for both activation and imaging. The cells were

113 then moved to the next position, and the process was repeated until the volumetric image was

114 collected. Each imaging volume consisted of 131 planes, which took $2.6 \mathrm{~s}$ to collect. After

115 optical activation, a significant aggregation of CRY2oligo-mRuby3 in the middle area of the

116 cells illuminated by the Bessel beam could be found (Figure 1e and Movie 1). Since the sample

117 scan mode was used, lines of mRuby3 fluorescence signals could clearly be seen at the center

118 of the cells. The diffusion of CRY2oligo-mRuby3 into the cytoplasm caused a broadening of

119 the width of the observed CRY2oligo-mRuby3 fluorescent strips over time. The intensity

120 profiles of different Bessel beams formed by various maximum and minimum NAs in addition

121 to the corresponding time-lapse MIP images of cell responses at the excitation laser power of

$1221 \mathrm{nW}\left(\sim 24 \mathrm{~mW} / \mathrm{cm}^{2}\right)$ are shown in Figure S3 of the Supplementary Information. Note that the

123 minimum excitation power at the focal plane required to produce a visible clustering signal

124 was $0.2 \mathrm{nW}$, which was low enough for a long-term living cell experiment. Before stimulation, 
126 LLSM could not trigger the clustering of the CRY2olig-mRuby3 protein (row 1 of Figures 1e).

127 We also tested the activation using different wavelengths $(405,445,488$, and $514 \mathrm{~nm})$ at $1 \mathrm{nW}$.

128 The activation of CRY2olig-mRuby3 was observed for all of the tested wavelengths (Figure

129 S4 and Movie 2 in the Supplementary Information).

130 3D activation using shifting Bessel beams

131 A Bessel beam is a non-diffracted beam in which the energy concentrates within its length and

132 diverges rapidly, as shown in figure S1. Taking advantage of this property, we demonstrated

133 that it was possible to shift the lattice beam for fast and large volumetric imaging, during which

134 the lattice beam could be shifted in the propagation direction by imposing a defocusing phase

135 profile on the $\mathrm{SLM}^{27}$. Therefore, it may be possible to create a Bessel beam that can locally

136 activate molecules in the beam propagation direction. In the previous section, we demonstrated

137 that the Bessel beam could activate the CRY2oligo-mRuby3 at a specific location in each 2D

138 frame during volumetric imaging where the contineous activation at the same postion in

139 diffrernt frames by Bessel beams acted as a Bessel fan activation on the $Y^{\prime} Z^{\prime}$ plane within one

140 volume scan (Figure 2a, Bessel fan). The corresponding timing diagrams for switching on the

141 activation (blue) and imaging (green) laser by two-color SLM and the movement of the sample

142 stage (black) are illustrated at the bottom of figure 2a. To control the activation in the beam

143 propagation direction, we generated a shifted Bessel fan in the Y direction via SLM with the

144 phase map shown in Figure 1b. The shift distance could be adjusted by changing the amplitude 
145 of the modulation phase profile on the SLM to locally activate the basal area of the cell (Figure

146 2a, shifted Bessel fan) with the defocusing Bessel pattern for activation using the same timing

147 diagram. Projection images of the cells activated by the Bessel fan activation across or

148 underneath the cell body at different time points are shown in Figure $2 b$ (movies 3 and 4). The

149 depth color image was used to illustrate the clustering of molecules and the cell edge. For the

150 regular Bessel fan activation, the XZ' and $Y^{\prime} Z^{\prime}$ projections (first row in Figure $2 \mathrm{~b}$ ) indicate that

151 the clustering CRY2oligo-mRuby3 initially took place $3 \mathrm{~s}$ after stimulation in the middle of the

cell. In contrast, the clustering of CRY2oligo-mRuby 3 was observed $21 \mathrm{~s}$ after activation at the

bottom of the cell when a shifted Bessel fan was used (second row in Figure 2b).

a volume image of the cell without activation to select the proper frame for activation. The

157 given frame within a 3D stack scan (Figure 2a single Bessel Beam) where the timing diagram

158 indicates the activation beam is switched on once at a specific $\mathrm{z}$ step during volumtric imaging.

159 Unlike the Bessel fan stimulation in which the cells were stimulated in every frame, the single

160 Bessel beam stimulation only activated the cells once per volume scan. The MIP images of the

161 cells activated by such single Bessel beam stimulation are depicted in Figure 2c, in which the

162 clustering of CRY2oligo-mRuby3 in the illuminating region could clearly be seen $21 \mathrm{~s}$ after 
164 from the illuminated area (marked as a white cross in Figure 2c and Movie 5). To analyze the

165 spatiotemproal behavior of the activated clusters, 3D cell volume renderings with selected

166 CRY2oligo-mRuby3 clusters are shown in Figure 3a, where cluster 1 is located right at the

167 activation region, clusters 2 and 3 are $2 \mu \mathrm{m}$ and $5 \mu \mathrm{m}$ away from the activation region,

168 respectively. The time-dependent fluorescence intensity changes of the chosen clusters are

169 plotted in Figure 3b, where cluster 1 shows an instantaneous increase upon the activation beam.

170 The delayed responses are observed according to the distance from the activation spot, for

171 example, $10 \mathrm{~s}$ for cluster 2 and $40 \mathrm{~s}$ for cluster 3 . To evaluate the photobleaching effect in our

172 system, we monitored the fluorescence signal of a pre-existing cluster (cluster 4), which was

173 far away from the activation beam. No decrease in fluorescence intensity of cluster 4 was

174 observed during the whole experimental period indicating that the photobleaching effect of our

175 system is negligible. A series of raw images for the selected plane activated by a single Bessel

176 beam are shown in Figure 3c, where the formation of the cluster over time is marked in a white

177 box. The analysis of fluorescence intensities of different clusters activated by Bessel fan and

178 shifted Bessel beam is shown in Figures S5 and S6. These findings clearly demonstrate that

179 LLSM could be easily modified to conduct 3D optogenetic experiment with good spatial

180 control using a regular and modified Bessel fan/beam as an activation source.

181 Subcellular optogenetic activation of membrane ruffling

182 The next challenge of using LLSM for optogenetic application was to induce optogenetic 
183 responses that had subcellular resolution. To control cellular responses at the subcellular level,

184 we adapted the CRY2-CIBN system to trigger the regional cell membrane ruffling. In this

185 experiment, the CRY2 was fused with PI3K-catalyzed motif (p85, iSH), which could trigger

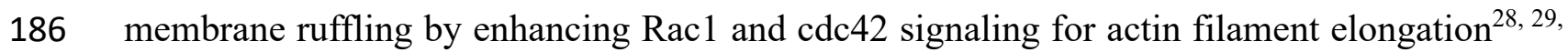

$183^{30}$, whereas a plasma membrane-anchor peptide (CAAX motif) was attached to CIBN. To

188 observe membrane ruffling, F-tractin-mCherry was used to monitor cytoskeletal dynamics. A

189 2A-self cleaved peptide (p2a) was used to connect each component, including F-actin-mCherry,

190 CRY2-iSH, and CIBN-CAAX to ensure nearly equal expression levels in the cell. We first

191 tested CRY2-mCherry-iSH-p2a-CIBN-EYFP-CAAX transfected cells using a Bessel fan

activation at $488 \mathrm{~nm}$ to trigger the response in the selected areas. The MIP images of CRY2-

193

mCherry in the cell in XY' plane are shown in Figure S7 and Movie 6 in the Supplementary

194 Information. To demonstrate that membrane ruffling can be induced with subcellular resolution,

195 two areas within a cell that were separated by $15 \mu \mathrm{m}$ were illuminated sequentially by the

196 activation beam (regions 1 and 2 in Figure S7), and membrane ruffling at each location could

197 clearly be seen after stimulation. To evaluate whether the function of the cells was

198 compromised by the activation process, we waited 30 min for the cells to recover to the non-

199 activated state (as shown in Movie 6). A lattice beam at $488 \mathrm{~nm}$ instead of a Bessel fan was

200 used to activate the whole cell. Membrane ruffling was observed over the whole-cell, unlike

201 the local membrane ruffling shown in Figure S7, indicating the function of the cell remained 
202 the same even after activation by the Bessel fan in two subcellular locations. Since CRY2

203 moved from the cytosol to the membrane, the detailed motion of membrane ruffling was not

204 clearly resolved in the volumetric images. To observe the detailed motion during membrane

205 ruffling, we studied the distribution of filamentous actin in the cells expressing F-tractin-

206 mCherry-p2a-CRY2-mCFP-iSH-p2a-CIBN-EYFP-CAAX. After stimulation (white cross in

207 figure 4a region 1), the membrane ruffled around the area under stimulation and moved inward

208 at the speed of $\sim 1 \mu \mathrm{m} / \mathrm{sec}$ where the moving distance of the membrane edge along the white

209 arrow is plotted in the inset. To demonstrate the capability of controlling the location of the

210 stimulation area, the stimulation beam was moved to the newly formed membrane (white cross

211 in figure 4a region 2) by shifting the Bessel beam, which was achieved by changing the SLM

212 pattern with the $\mathrm{X}$-shift as shown in Figure 1b. The magnitude of membrane ruffling at region

2132 was larger than at region 1 (detailed membrane ruffling can be found in Movie 7). To present

214 the dynamics of the filament actin, we used color-coded signals at different time points, as

215 shown in Figure 4a. To better illustrate the dynamic behavior of the filamentous actins (the

216 enlarged part of figure 4a), the contour function of the Amira software was used to outline the

217 shape of the cell using the fluorescent signal from F-tractin-mCherry. The membrane protrusion

218 and retraction upon the activation, as shown in Figure S8, reveals that the membrane ruffling

219 is presumably increased at the site of activation as compared to the rest of the cell body.

220 However, after the second stimulation at the newly formed protrusion (region 2), the membrane 
221 ruffled rapidly in 3D at the edge of the cell as seen by the projection images in XY' and XZ' of

222 long-range actin movements (Figure 4a). These results demonstrate that our system is capable

223 of monitoring and activating the optogenetic molecules at the subcellular level.

\section{Long-term manipulation of cell migration on lattice lightsheet microscope}

225 To validate the capability of our technique with respect to manipulating cellular behavior for

226 a long period of time, we first established an optogenetic system to control the migration of a

227 cell in which the epidermal growth factor receptor (EGFR) was fused with CRY2olig and three

228 repeating mApple (EGFR-CRY2olig-mApplex3) to activate the intracellular signaling of

229 EGFR through blue-light induced clustering ${ }^{31,32}$. By maximizing the brightness of red

230 fluorescence protein for whole-cell imaging, the cross-talk activation could be minimized at a

231 very low laser power. EGFR activation is known to trigger cell migration through the PI3K

232 signaling ${ }^{33}, 34$. When the cells expressing EGFR-CRY2olig-mApplex3 were activated by the

233 Bessel fan in the trailing edge, the formation of lamellipodia in the trailing edge was observed,

234 as shown in Figure 4b. The distribution of EGFR is color-coded at different time points. From

235 Figure $4 \mathrm{~b}$ and Movie 8 , it can be seen that the direction of migration was altered after

236 stimulation in which the formation of lamellipodia was observed in the original trailing edge,

237 and the old leading edge had started to retract. These results confirmed that the direction of

238 migration could be controlled by optically induced clustering of the EGFR-CRY2olig- 
240 Next, a directed migration experiment was conducted by continuous stimulation. Since the cell

241 would migrate out of the field of view, the position of the samples every 20-30 minutes was

242 adjusted while maintaining the same stimulated area in the cell, as shown in Movie 9. To

243 illustrate the trajectories of the cell in the same image, we marked the boundary of the cell at

244 each time point with different colors. The time-dependent trajectories of the cell expressing

245 EGFR-CRY2olig-mApplex3 stimulated by a Bessel fan in LLSM are shown in Figure 5. The

246 individual LLSM rendering images of the 3D cell and the stimulated area are depicted at the

247 bottom of Figure 5 and Movie 10. Moreover, the volumes of the migrating cell over time are

248 calculated and plotted in the inset, where the volume of cell reduces when migration direction

249 cahnges at $\mathrm{T}=265$, followed by the formatio of large lamellipodia at $\mathrm{T}=292$. From these results,

250 we demonstrated that the combination of LLSM and Bessel fan stimulation was useful for

251 continuously manipulating and monitoring EGFR-CRY2olig-mApplex3-expressing cells for

252 more than 6 hours, where $\sim 75,000$ 2D-images (or 463 volumetric images) were collected, a

253 finding that introduces the opportunity for conducting long-term optogenetic experiments in

254 living cells.

255 Discussion

256 In optogenetic LLSM experiments, an additional stimulating light source is used. Therefore,

257 we would like to evaluate the energy level used in the activation process. The minimum energy 
259 that the minimum energy required to induce the clustering of CRY2olig-mRuby3 molecules

260 was $0.2 \mathrm{nW}$ at $488 \mathrm{~nm}$, and $1 \mathrm{nW}$ was enough to activate the CRY2olig-mRuby3 system with

261 a Bessel beam at 405, 445, 488, and $514 \mathrm{~nm}$. For volumetric imaging in LLSM, the total power was $40 \mathrm{nW}$ at $561 \mathrm{~nm}$, which was distributed over the 145 lattice beams for the collection of

fluorescent images with a signal-to-noise ratio greater than 5 for the observations of CRY2,

CRY2oligo, filament-actin, and EGFR.

Bessel beams with a minimized energy distribution in the sidelobes. However, when a single

Bessel beam is used as the activation source, the influence of the energy distribution in the

sidelobes, which could deteriorate the spatial resolution for activation, should be considered. A

271 optogenetic clustering of CRY2olig-mRuby3 using different Bessel beams formed by various

272 minimum NAs with the same maximum NA was evaluated. The energy distributions of the

273 Bessel beams at the back aperture and focal planes (XZ) can be found in the first and second

274 row of Figure S3a of the Supplementary Information.

275 The LLSM images of the optogenetic clustering in U2OS cells expressing CRY2olig-mRuby3

276 stimulated by different Bessel fans at different time points are found in the fifth to the eighth

277 row. Since the raw LLSM images contained all signals from CRY2olig-mRuby3, the Threshold 
278 function in the Amira software (Thermo Fisher Scientific) was selected to calculate the spatial

279 distribution of the optogenetic clustering (an example is shown in Figure S3b). The measured

280 width of the Bessel fan and the width of optogenetic clustering are depicted in Figure S3c. It was observed that the spatial distributions of the optogenetic clustering were much wider than

282 the width of the stimulating Bessel beams, with ratios ranging from 4 to 5 . The measured Bessel beam profiles in dye solution using different numerical apertures (NAs), as shown in Figure S3, also confirmed the agreement between the simulations and the experimental data. To characterize the Bessel fan and single Bessel beam activation, we measured the fluorescence signals of the photoactivated CRY2oligo-mRuby 3 molecules by $488 \mathrm{~nm}$ in figure S12 (movies used to indicate the beam profile of the photoactivation beam. In the Bessel fan activation, a 
297 original state at $1 \mathrm{nW}$ stimulation. The energy used for CRY2Olig-mRuby3 activation was

298 similar to the CRY2-CIBN dimerization energy. To control cell migration, CRY2Olig was

299 linked to the intracellular part of the EGFR, in which optically-induced oligomerization of

300 CRY2Olig led to EGFR oligomerization. It is known that the existence of other EGFR

301 molecules in close proximity with each other will promote EGFR signal activation. Therefore,

302 the direction of migration could be reversed by activating EGFRs in the trailing end of the

303 migrating cells, as shown in Figures $4 \mathrm{~b}$ and 5.

304 To achieve cell manipulation in 3D, a focus shift was used in this experiment in which regions

305 illuminated by the light sheet could be controlled by adding a binary phase Fresnel lens on the

306 spatial light modulator used to generate Bessel beams filtered by a passive optical component,

307 physical mask. As shown in figure 1b, the region illuminated by the light sheet, where its length

308 and thickness are pre-determined by a chosen annual mask, could be moved forward or

309 backward in the light propagation direction. Within the length of the lightsheet, the optogenetic

310 system could be activated effectively, whereas the energy distribution of the lightsheet diverged

311 rapidly beyond the length of the light sheet but was not enough for optogenetic system

312 activation (energy distribution is shown in figure S2). Therefore, the optogenetic molecules

313 located on the membranes could be activated locally at the apical or basal membranes to

314 achieve a controllable optogenetic system in 3D. Despite the contribution from the sidelobes

315 of a Bessel stimulation, which can be minimized by carefully engineering the beam shape 
316 before the experiment as shown in figure S12, not only the diffusion effect of CRY2oligo-

317 mRuby3 but also the energy distribution in the sidelobes still contributed to the broadened

318 width in the activated area as shown in figure S3c. To improve the spatial confinement for

319 activation, two-photon activation or temporal focusing may be used ${ }^{35,36}$. However, the

320 integration of these techniques into LLSM may be complicated. Another challenge in

321 implementing 3D activation in LLSM was the line confinement activation instead of a point in

322 the propagation direction, which was about $20 \mu \mathrm{m}$ at the present configuration in order to cover

323 the whole cell imaging. Such confinement allows us to condcut local activation of molecules

324 located on the apical or basal membrane by SLM manipulation but not enough to activate

325 molecules within a specific organelle in the cells with the fixed annual mask. An aperture-free

326 technique for the generation of activation beam and lattice beam at different beam lengths is

327 required in the future. In summary, the 3D activation capability of our appraoch opens the

328 opportunity for optogenetic activation of single cells in small animal models. 


\section{Materials and Methods}

\section{Plasmid construction}

333 For the multiple-gene expression system, the plasmid pCR3.1-p2a-SpeI was generated from

334 the original pCR3.1 plasmid, which was added in-frame to the porcine teschovirus-12A self-

335 cleaving peptide (p2a) and SpeI cutting site to fit with the NheI cutting site of the enhanced

336 green fluorescent protein (EGFP) series of vectors. The F-tractin-mCherry-p2a-SpeI, CRY2-

337 mCherry-iSH-p2a-SpeI and CRY2-mCFP-iSH-p2a-SpeI were constructed based on pCR3.1-

338 p2a-SpeI plasmid. The filamentous actin marker (a cytoplasmic actin filament reporter, the

339 neuronal inositol 1,4,5-triphosphate 3-kinase A actin-binding domain known as F-tractin

340 [Plasmid \#58473, addgene]) was fused with monomeric red fluorescent protein to the N-

341 terminal p2a peptide via digestion using two restriction enzymes, NheI-HF and PmlI, and

342 ligated into pCR3.1-p2a-SpeI plasmid. Using the specific primer polymerase chain reaction

343 (PCR) cloning, the C-terminal of Arabidopsis cryptochrome 2 (CRY2) with mCherry or mCFP

344 (monomer cyan fluorescent protein) was linked with the phosphoinositide 3 kinase (PI3K)-

345 catalyzed motif (iSH) to generate CRY2-mCherry-iSH-p2a-SpeI and CRY2-mCFP-iSH-p2a-

346 SpeI plasmids. The multiple gene expression of the backbone plasmid, which contains the

347 CRY2 interaction binding protein N-truncated version (CIBN) domain linked to the plasma membrane anchor leading sequence (CIBN-CAAX), was cut by double digestion using NdeI 
351 same protocol for generating CRY2-mCherry-iSH-p2a-CIBN-EYFP-CAAX and F-tractin-

353 hand, the generation of CRY2olig-mRuby3. CRY2olig-mCherry plasmids were modified by

354 point mutation PCR to generate an XmaI cutting site for compatible cohesiveness with the AgeI

355 cutting site. The CRY2olig-XmaI-mCherry plasmids were then digested with XmaI and BsrGI.

356 Using an AgeI and BsrGI double digestion, the mRuby3 was inserted into the CRY2olig-XmaI

357 plasmid. To prepare CRY2Olig-mApplex3, the BsrGI-mApple-BsiWI-PmlI was first created

358 by specific primer PCR to increase the repeat of mApple, and AgeI and BsiWI (compatible

359 cohesive end with BsrGI) were used to replace the original mRuby3 site of CRY2Olig-mRuby3.

360 The XhoI cutting site of EGFR fits in the frame with the open reading frame of CRY2Olig-

mApplex3. EGFR was cut with NheI and XhoI for insertion into the N-terminus of CRY2Olig-

mApplex3 for creating EGFR-CRY2Olig-mApplex3.

\section{Imaging of optogenetic tools expressed cell line}

364 U2OS cells were maintained in Dulbecco's modified Eagle's medium (DMEM, Gibco,

365 ThermoFisher), which contained 10\% fetal bovine serum (FBS) from Gibco (ThermoFisher),

$3662 \mathrm{mM}$ L-glutamine (Gibco, ThermoFisher), and 1\% penicillin-streptomycin solution (Gibco,

367 ThermoFisher). Before transfection, $5 \mathrm{~mm}$ glass coverslips were placed in $1 \mathrm{X}$ phosphate-

368 buffered saline (PBS) buffer with $10 \mu \mathrm{g}$ fibronectin for $2 \mathrm{~h}$ at room temperature. The

369 fibronectin-coated coverslips were washed twice with 5X PBS and once with 1X PBS. U2OS 
370 cells $\left(1 \times 10^{5}\right)$ were seeded into 12-well plates containing the fibronectin-coated glass

371 coverslips and cultured in DMEM medium without penicillin-streptomycin to enhance

372 transfection efficiency.

373 Cells were incubated for $16 \mathrm{~h}$ at $37^{\circ} \mathrm{C}$ and $5 \% \mathrm{CO}_{2}$ before undergoing transfection with $0.8 \mu \mathrm{g}$

374 plasmid DNA per well using Maestrofectin Transfection Reagent (Omics Bio). After $16 \mathrm{~h}$, the

375 transfected cells were allowed to recover for $24 \mathrm{~h}$ in $1 \%$ penicillin-streptomycin and $10 \%$ FBS

376 DMEM medium.

377 Stimulation setting and Image acquisition

378 In order to standardize the stimulation conditions, we used a power meter (PD300-1W with

379 Nova II, Ophir Photonics, USA) to detect the input power of the Bessel beam behind the pupil

380 of the exciting objective. The required parameters for different wavelengths $(405,445,488$,

381 and $514 \mathrm{~nm})$ and power $(0.5,1,2$, and $4 \mathrm{nW})$ were adjusted according to the readings. To

382 measure the laser power below the detection limit of the power meter, which is about $1 \mathrm{nW}$, the

383 laser power was attenuated linearly by a combination of the acousto-optic tunable filter (AOTF)

384 and the neutral density filters. Multi-color scanning was used in the Z-scan mode in the SPIM

385 program (licensed by Howard Hughes Medical Institute, Janelia Research Campus) to generate

386 multi-color 3D images, on which the signals from each channel were collected one by one from

387 the same layer. As shown in the bottom of figure 2a, the SLM displaying two pre-loaded color 
each step of the sample stage at the designed time sequence based on the experimental exposure

390 time in the SPIM software. In an optogenetic experiment, the excitation source was first

391 switched to the projected Bessel beam mode for stimulation and then followed by lattice beams

392 for imaging. In each experiment, blank control images were collected for the first 20 time points

393 by turning off the stimulating light source with a mechanical shutter which is placed in front of

394 the $488 \mathrm{~nm}$ laser, controlled by a homemade Arduino device synchronized to the image

395 acquisition software. For single Bessel beam stimulation, the SPIM program held the $\mathrm{x}-$

396 galvanometer without any movement, thus allowing the Bessel beam to stimulate the central

397 area of the cells, and the activation wavelength $488 \mathrm{~nm}$ was illuminated on the samples at a

398 selected $\mathrm{z}$ slice while performing z-stacking; the timing is clocked by Arduino device.

399 In the experiment requiring moving the stimulation area along the $\mathrm{X}$-axial direction, the

400 position of the Bessel pattern on the SLM could be changed to illuminate the corresponding

401 focal plane position. Alternatively, the x-galvo could be used to move the illumination area of

402 the Bessel beam to a specified position with an accuracy of $0.1 \mathrm{um}$. In the single-Bessel beam

403 stimulation experiment, a shutter was used to allow the stimulation beam to activate the cells

404 once per volume scan. In addition, the tiling lattice lightsheet technique was used in the

405 experiment to shift the Bessel beam along the Y-axial direction by changing the beam shift

406 coefficient as described previously ${ }^{27}$.

\section{Image capturing and processing}


408 The details for the LLSM optical scheme can be found in the supplementary note. In this

410 in which an area of $200 \times 960$ pixels $(20.6 \mu \mathrm{m} \times 98.9 \mu \mathrm{m})$ was used for LLSM imaging. In a

411 typical LLSM imaging setup, the exposure time for each frame is $9 \mathrm{~ms}$, the sample scanning

412 step is $0.6 \mu \mathrm{m}$, and 131 planes of images are used for volumetric imaging. For time-lapse

413 volumetric imaging, the volumetric image is recorded every $3 \mathrm{~s}$. The camera settings for the

414 directed cell migration experiment are the same (10 ms exposure time for $200 \mathrm{x} 1024$ pixels with 161 planes) for both colors $488 \mathrm{~nm}$ (activation) and $560 \mathrm{~nm}$ (imaging), where it takes $~ 3$ $\mathrm{s}$ to finish 3D optogenetic imaging. Initially, the time interval between each volumetric image was set to $6 \mathrm{~s}$ until we observed cellular response or membrane ruffling (in this case, 60-time points). We then changed the time interval to $1 \mathrm{~min}$ to observe directed cell migration for 343-

419 time points. Note that for cell manipulation, such as induced membrane ruffling and directed

420 cell migration experiments, the subcellular responses of cells cannot be predicted. Therefore,

421 we cannot program the SLM pattern time profile in advance. To demonstrate the capability of

422 local activation, the photoactivation was conducted manually. After recording the first cellular

423 response, the program was stopped, and the activation beam was moved to the desired area

424 either by changing the SLM pattern or the x-galvo position. The program was resumed to record

425 the cellular response. These processes were repeated until the end of the experiment. Since the 
427 the coverslip with a view angle similar to conventional microscopy were reconstructed from

428 the raw images through the GPU program. To process the images for better presentation in

429 Figures 4 and 5, Amira imaging process software (ThermoFisher) was used. For the directed

430 cell migration, the timing and position were registered in the movie reconstruction, as shown

431 in figure 5 and movie 10.

432

433

434

\section{Acknowledgment}

436 The microscope control software is licensed by the Howard Hughes Medical Institute, Janelia

437 Research Campus. This work was supported by the Ministry of Science and Technology,

438 Taiwan (Project No. MOST 109-2113-M-001 -034 -MY3 to P.C., 109-2628-M-001-001-MY4,

439 110-2321-B-002-012 to B.C.C., MOST 110-2636-B-007-011, and MOST 108-2638-B-010-

440 001-MY2 to Y.C.L.). This work was also supported by the Academia Sinica, Taipei, Taiwan

441 (AS-IA-110-M04 to P.C. and AS-CDA-107-M08 to B.C.C.). We also thank the DNA

442 Sequencing Core Facility of the Institute of Biomedical Sciences, Academia Sinica, for DNA

443 sequencing analysis. The core facility is funded by Academia Sinica Core Facility and

444 Innovative Instrument Project (AS-CFII-108-115). 


\section{Figure Legends}

$449 \quad$ Figure 1

450 (a) The schematic diagram of the major optical components in lattice lightsheet microscopy 451 (LLSM). The patterns displayed by the spatial light modulator (SLM) are imaged at the pupil 452 plane of the excitation objective through an annual mask and lens. Lattice beams, in addition 453 to Bessel beams with different X, Y positions, can be generated at the imaging plane by 454 imposing different patterns on the lightsheet microscopy (LSM). (b) Various patterns on the 455 SLM for generating the corresponding excitation beams in the imaging plane. The position of 456 the Bessel beams can be shifted in the X or Y direction by using X- or Y-shift patterns. For 457 normal Bessel beam stimulation, the Bessel beam with a length of $20 \mu \mathrm{m}$ illuminates the cell 458 at a tiled angle of 32.8 degrees. With Y-shift stimulation, the Bessel beam can be moved along 459 the propagation direction to illuminate the basal membrane. With an X-shift pattern, the Bessel 460 beam can be moved to a different location in the $\mathrm{x}$-direction with an accuracy that is better than $461100 \mathrm{~nm}$. (c) The illumination scheme for an optogenetic experiment. The blue beam represents 462 the Bessel beam at $488 \mathrm{~nm}$ used to activate the optogenetic molecules, and the green lightsheet 463 represents the $561 \mathrm{~nm}$ lattice lightsheet for imaging. The volumetric image was obtained by 464 dithering the lattice beam and moving the sample along the $Y^{\prime}$ direction. (d) The schematic for 465 optical induced clustering of cryptochrome 2 (CRY2)olig-mRuby3. Blue: CRY2olig, Red: 466 mRuby3. (e) The time-lapse maximum intensity projection (MIP) images of CRY2olig467 mRuby 3 expressed cells with various activation energies. The interval between each stack is 3 468 s. Scale bar $10 \mu \mathrm{m}$.

\section{$470 \quad$ Figure 2}

471 (a) The schematic for various activations: Bessel fan, shifted Bessel fan, and single Bessel 472 beam. The activation beam illuminates on the $Y^{\prime} Z^{\prime}$ plane at an angle of $32.8^{0}$. Bottom: The 473 corresponding timing diagrams for the SLM patterns with laser $488 \mathrm{~nm}$ Bessel beam (blue) and $474560 \mathrm{~nm}$ lattice beam (green) illumination synchronized with sample piezo waveforms (black). 475 (b) The time-lapse images of optical induced clustering of CRY2oligo-mRuby3 in XY' Y'Z' 476 XZ' planes stimulated by Bessel fan and shifted Bessel fan activation. The depth of the 477 oligomerization is color-coded, where the process of oligomerization occurs only at the basal 478 membrane by the shifted Bessel beam activation. (c) The center part of the cell marked by the 
479 white cross is illuminated by the single Bessel beam. Oligomerization occurs near the activation site. Scale bar $10 \mu \mathrm{m}$.

\section{Figure 3}

483 (a) Top view and side view of the 3D cell volume rendering image of the selected clusters of CRY2oligo-mRuby3 molecules induced by a single Bessel beam activation at the spot shown in Figure 2c. Scale bar $10 \mu \mathrm{m}$. (b) Time-lapse fluorescence intensity plots of the marked clusters in (a). (c) Left: Raw image of the optical induced clustering of CRY2oligo-mRuby3 in the selected plane activated by a Bessel beam marked in a white cross in Fig. $2 \mathrm{c}$ at $\mathrm{T}=120 \mathrm{~s}$. Right: Enlarged raw time-lapsed images of the green box in the left in the single Bessel beam activation experiment. Scale bar $10 \mu \mathrm{m}$ and $1 \mu \mathrm{m}$, respectively.

490

491

492

\section{Figure 4}

493 (a)The cells expressing F-tractin-mCherry-p2a-CRY2mCFPiSH-p2a-CIBNEYFPcaax are 494 illuminated by single Bessel beam activation. The cross represents the activation site. To 495 demonstrate the dynamic behavior of filamentous actin, intensity contours were plotted before and after the stimulation. At region 1, the distance of membrane edge along the white arrow is shown in the inset. Different color represents different time points after stimulation. The top images are $\mathrm{XY}$ orthographic view, and the bottom images are $\mathrm{XZ}$ view with $32.8^{\circ}$ rotation in $\mathrm{X}$-axis. The gray images are the original images. (b). The Bessel fan was used to stimulate the endothelial growth factor receptor (EGFR)-CRY2Olig-mApplex3-expressing cells at the trailing edge. The images in the center row are the whole-cell images at different times poststimulation. The stimulation areas are marked as cyan. The solid arrow indicates the original trailing edge, and the hollow arrow indicates the original leading edge. Images on the top and bottom rows are enlarged views of the dashed box area in the middle row. Different colors represent different time points. Scale bar $10 \mu \mathrm{m}$

\section{Figure 5}

508 The time-dependent trajectories of the cell in the guided migration experiment. The cell expressing EGFR-CRY2olig-mApplex3 was stimulated by 1nW Bessel fan. Different colors represent different time points. The individual LLSM 3D rendering images of the cell at

511 different times, and the stimulated areas are depicted at the bottom. The volume changes of the

512 cell during the cell-guided migration are plotted in the inset. The interval between each 513 stimulation and image is about $1 \mathrm{~min}$. The entire experiment lasted 392 minutes. Scale bar 20 $514 \mu \mathrm{m}$ 


\section{References}

517 1. Karunarathne WK, Giri L, Patel AK, Venkatesh KV, Gautam N. Optical control demonstrates switch-like PIP3 dynamics underlying the initiation of immune cell

520

521

2. Xu Y, et al. Optogenetic control of chemokine receptor signal and T-cell migration. Proc Natl Acad Sci U S A 111, 6371-6376 (2014).

523

524

3. Gao L, Shao L, Chen BC, Betzig E. 3D live fluorescence imaging of cellular dynamics

525

526

527

4. Chatterjee K, Pratiwi FW, Wu FCM, Chen P, Chen BC. Recent Progress in Light Sheet Microscopy for Biological Applications. App/ Spectrosc 72, 1137-1169 (2018).

529

530

5. Ronzitti E, Ventalon C, Canepari M, Forget BC, Papagiakoumou E, Emiliani V. Recent 531 advances in patterned photostimulation for optogenetics. Journal of Optics 19, (2017).

532

533

6. Allen J. Application of patterned illumination using a DMD for optogenetic control of 534 signaling. Nature Methods 14, 1114-1114 (2017).

535

536

7. Johnson HE, Goyal Y, Pannucci NL, Schupbach T, Shvartsman SY, Toettcher JE. The Spatiotemporal Limits of Developmental Erk Signaling. Dev Cell 40, 185-192 (2017).

8. Kim JM, Lee M, Kim N, Heo WD. Optogenetic toolkit reveals the role of Ca2+ sparklets in coordinated cell migration. Proc Natl Acad Sci U S A 113, 5952-5957 (2016).

541

542 9. Planchon TA, et al. Rapid three-dimensional isotropic imaging of living cells using Bessel beam plane illumination. Nat Methods 8, 417-423 (2011).

10. Chen $\mathrm{BC}$, et al. Lattice light-sheet microscopy: imaging molecules to embryos at high spatiotemporal resolution. Science 346, 1257998 (2014).

11. Liu Z, Lavis LD, Betzig E. Imaging live-cell dynamics and structure at the single-molecule level. Mol Cell 58, 644-659 (2015).

12. Lu CH, et al. Lightsheet localization microscopy enables fast, large-scale, and threedimensional super-resolution imaging. Commun Biol 2, 177 (2019). 
554

555

556

557

558

559

560

561

562

563

564

565

566

567

568

569

570

571

572

573

574

575

576

577

578

579

580

581

582

583

584

585

586

587

588

589

590

591

13. Keller PJ, et al. Fast, high-contrast imaging of animal development with scanned light sheet-based structured-illumination microscopy. Nat Meth 7, 637-642 (2010).

14. Chang B-J, et al. Two-beam interference lattice lightsheet for structured illumination microscopy. Journal of Physics D: Applied Physics 53, 044005 (2019).

15. Ritter AT, et al. Actin depletion initiates events leading to granule secretion at the immunological synapse. Immunity 42, 864-876 (2015).

16. Fritz-Laylin LK, et al. Actin-based protrusions of migrating neutrophils are intrinsically lamellar and facilitate direction changes. Elife 6, (2017).

17. Liu Z, et al. 3D imaging of Sox2 enhancer clusters in embryonic stem cells. Elife 3, e04236 (2014).

18. Pfisterer K, et al. FMNL2 regulates dynamics of fascin in filopodia. J Cell Biol 219, (2020).

19. Ducros $M$, et al. Lattice light sheet microscopy and photo-stimulation in brain slices. In: Neural Imaging and Sensing (2019).

20. Leyden $\mathrm{F}$, et al. Rac1 activation can generate untemplated, lamellar membrane ruffles. BMC Biol 19, 72 (2021).

21. Wang MJ, Artemenko Y, Cai WJ, Iglesias PA, Devreotes PN. The directional response of chemotactic cells depends on a balance between cytoskeletal architecture and the external gradient. Cell Rep 9, 1110-1121 (2014).

22. Tischer D, Weiner OD. Illuminating cell signalling with optogenetic tools. Nat Rev Mol Cell Biol 15, 551-558 (2014).

23. Benedetti L, Barentine AES, Messa M, Wheeler H, Bewersdorf J, De Camilli P. Lightactivated protein interaction with high spatial subcellular confinement. Proc Natl Acad Sci U S A 115, E2238-E2245 (2018).

24. Kennedy MJ, Hughes RM, Peteya LA, Schwartz JW, Ehlers MD, Tucker CL. Rapid bluelight-mediated induction of protein interactions in living cells. Nat Methods 7, 973-975 (2010). 
593

594

595

596

597

598

599

600

601

602

603

604

605

606

607

608

609

610

611

612

613

614

615

616

617

618

619

620

621

622

623

624

625

626

627

628

629

25. Taslimi A, et al. An optimized optogenetic clustering tool for probing protein interaction and function. Nat Commun 5, 4925 (2014).

26. Chen B-C. Scanless lattice light sheet microscopy. Microscopy and Microanalysis 21, 715-716 (2015).

27. Gao L, Tang WC, Tsai YC, Chen BC. Lattice light sheet microscopy using tiling lattice light sheets. Opt Express 27, 1497-1506 (2019).

28. Etienne-Manneville S. Cdc42--the centre of polarity. J Cell Sci 117, 1291-1300 (2004).

29. Yang HW, Collins SR, Meyer T. Locally excitable Cdc42 signals steer cells during chemotaxis. Nat Cell Biol 18, 191-201 (2016).

30. Idevall-Hagren O, Dickson EJ, Hille B, Toomre DK, De Camilli P. Optogenetic control of phosphoinositide metabolism. Proc Natl Acad Sci U S A 109, E2316-2323 (2012).

31. Bugaj LJ, Spelke DP, Mesuda CK, Varedi M, Kane RS, Schaffer DV. Regulation of endogenous transmembrane receptors through optogenetic Cry2 clustering. Nat Commun 6, 6898 (2015).

32. Park H, Kim NY, Lee S, Kim N, Kim J, Heo WD. Optogenetic protein clustering through fluorescent protein tagging and extension of CRY2. Nat Commun 8, 30 (2017).

33. Friedl P, Gilmour D. Collective cell migration in morphogenesis, regeneration and cancer. Nat Rev Mol Cell Biol 10, 445-457 (2009).

34. Inoue T, Meyer T. Synthetic activation of endogenous PI3K and Rac identifies an ANDgate switch for cell polarization and migration. PLoS One 3, e3068 (2008).

35. Hernandez O, Papagiakoumou E, Tanese D, Fidelin K, Wyart C, Emiliani V. Threedimensional spatiotemporal focusing of holographic patterns. Nat Commun 7, 11928 (2016).

36. Oron D, Tal E, Silberberg Y. Scanningless depth-resolved microscopy. Optics Express 13, 1468-1476 (2005). 
bioRxiv preprint doi: https://doi.org/10.1101/2022.01.02.474058; this version posted January 2, 2022. The copyright holder for this preprint (which was not certified by peer review) is the author/funder. All rights reserved. No reuse allowed without permission. 


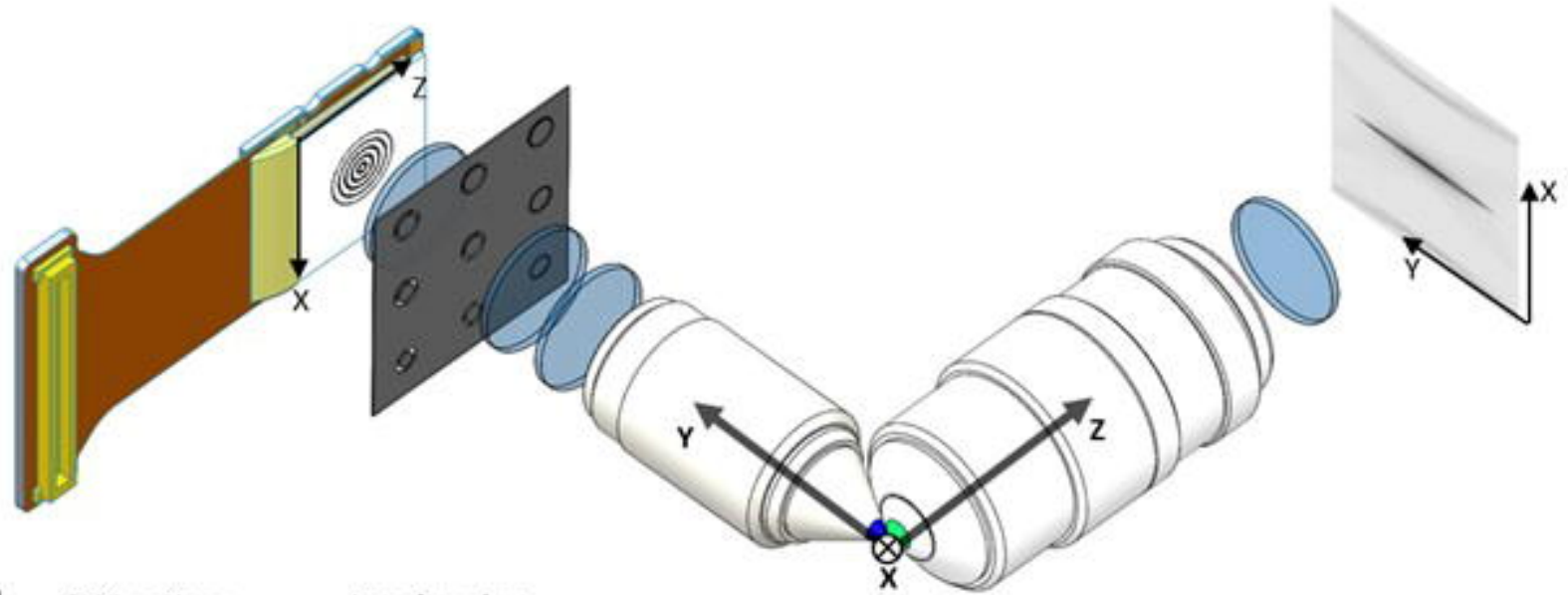

b

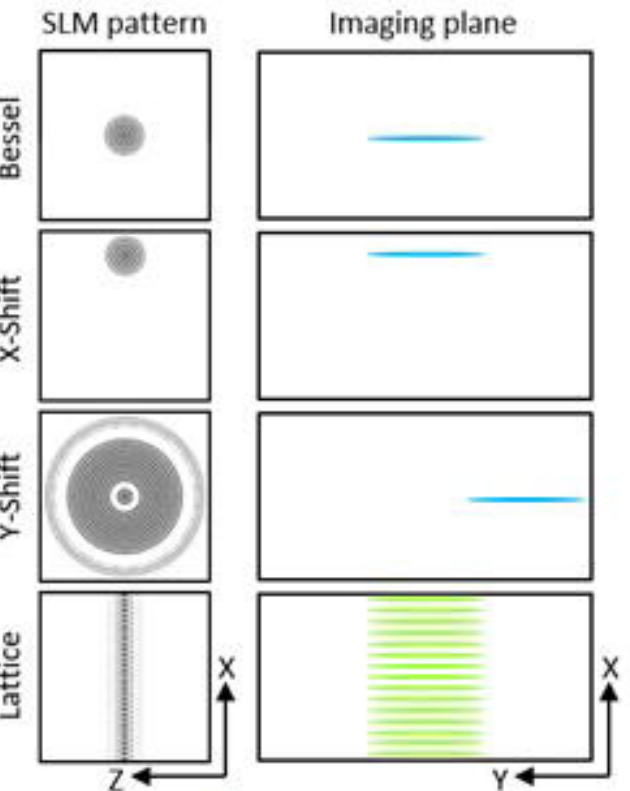

Bessel Beam

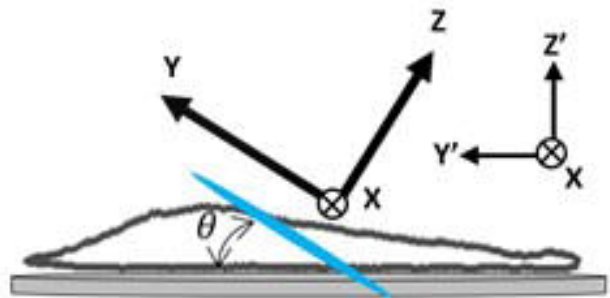

X-Axial Beam Shift

C

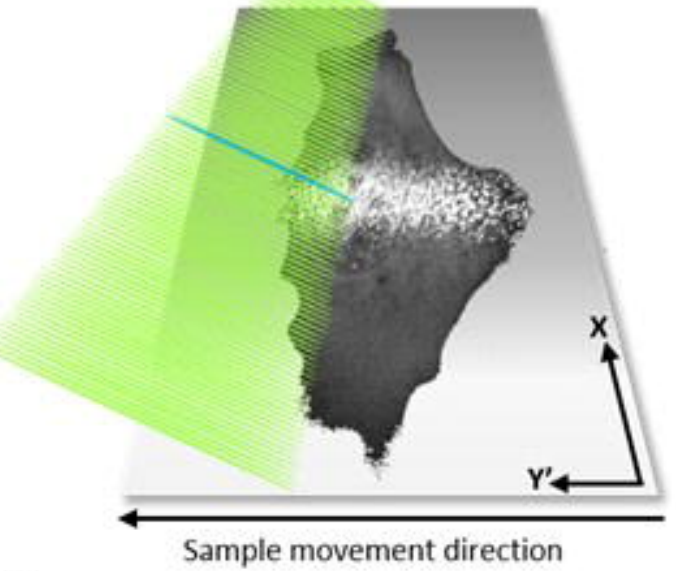

d

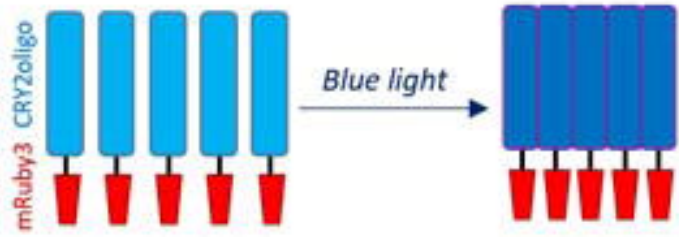

\section{Y-Axial Beam Shift}

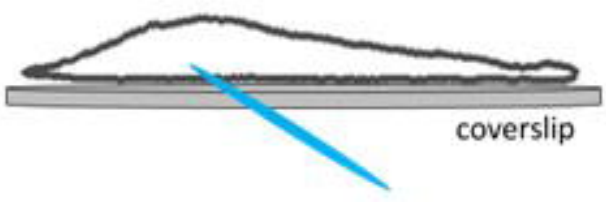

e

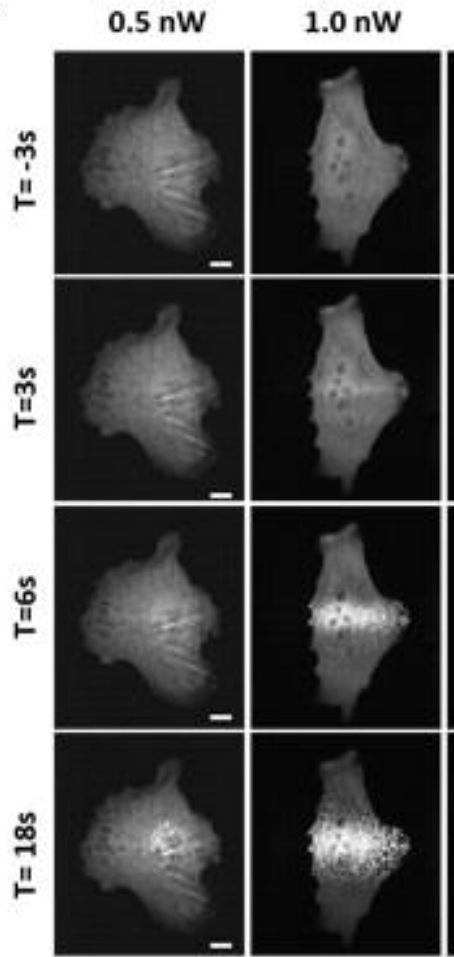

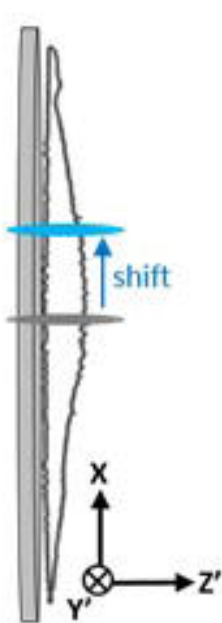

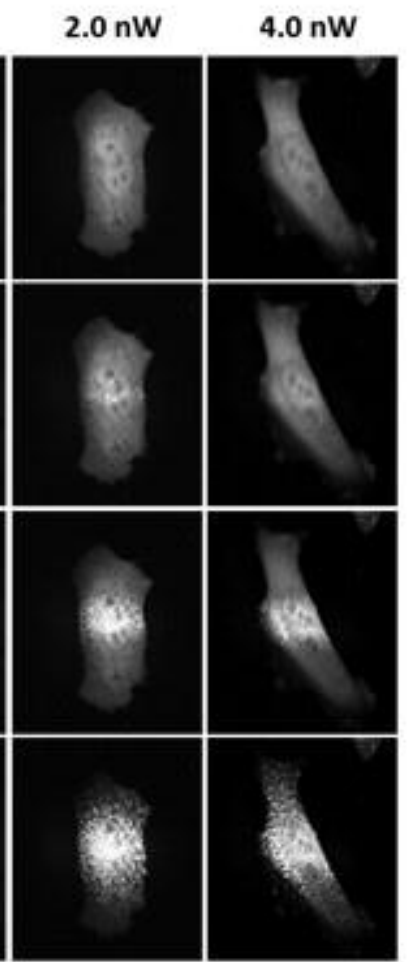




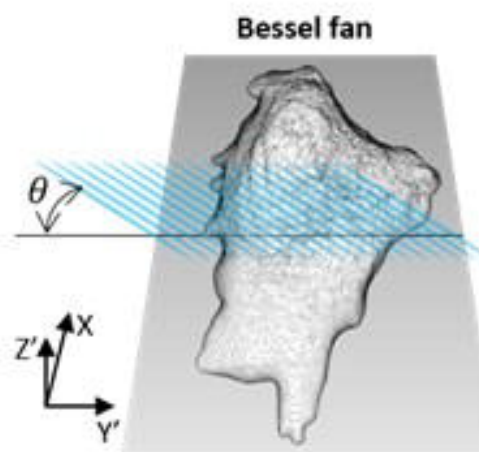

shifted Bessel fan
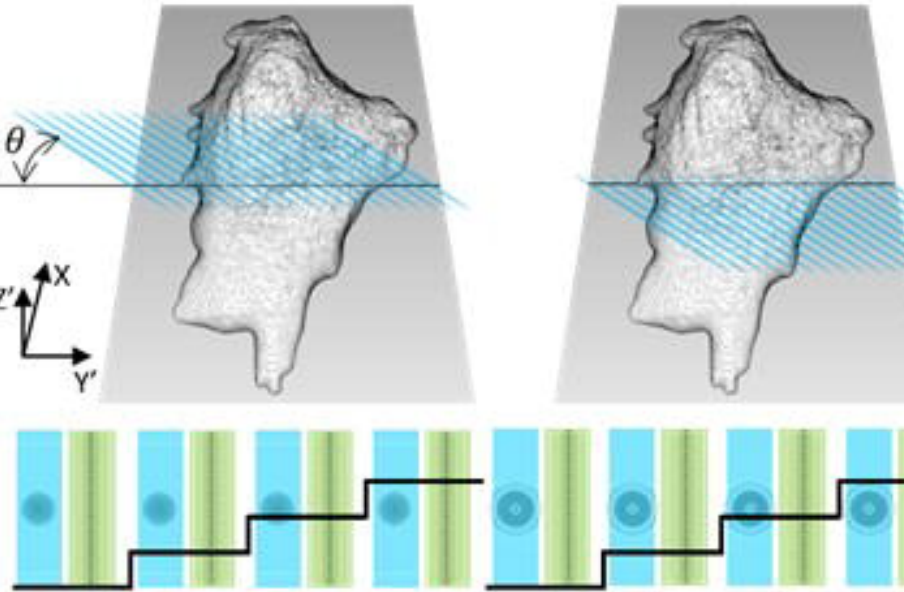

b
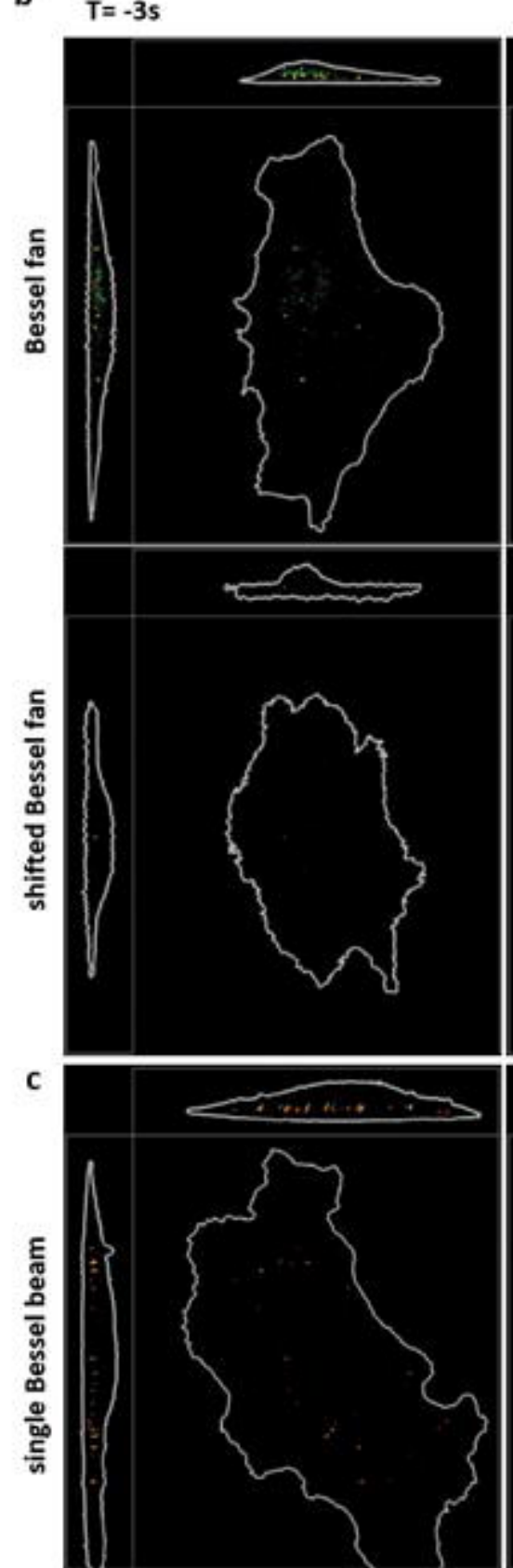

$T=3 s$
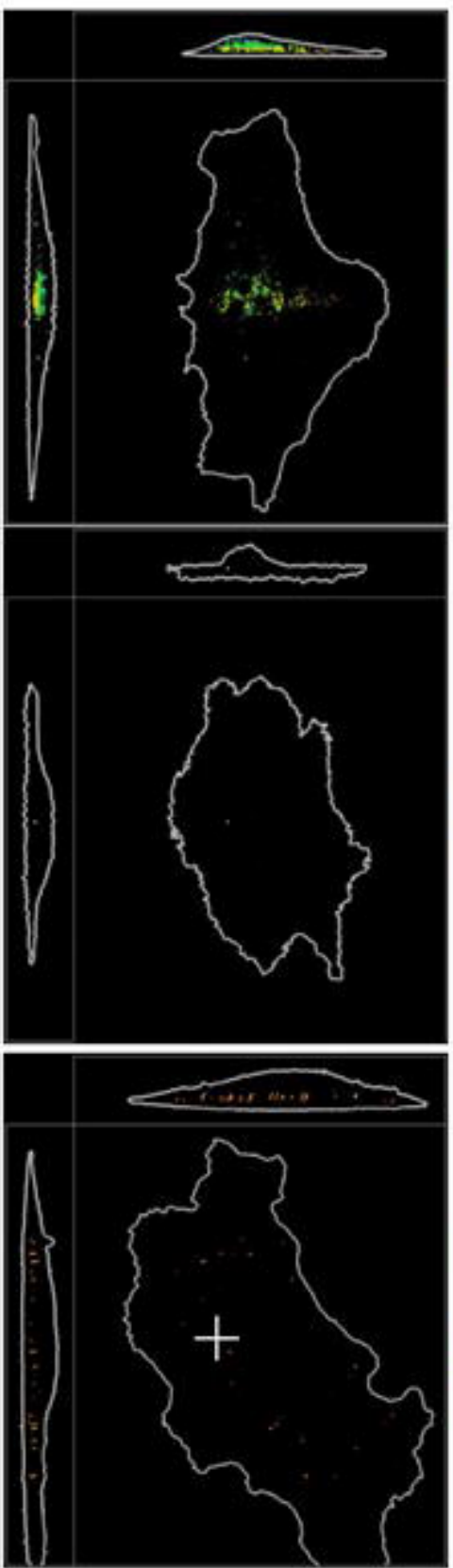

$T=21 \mathrm{~s}$

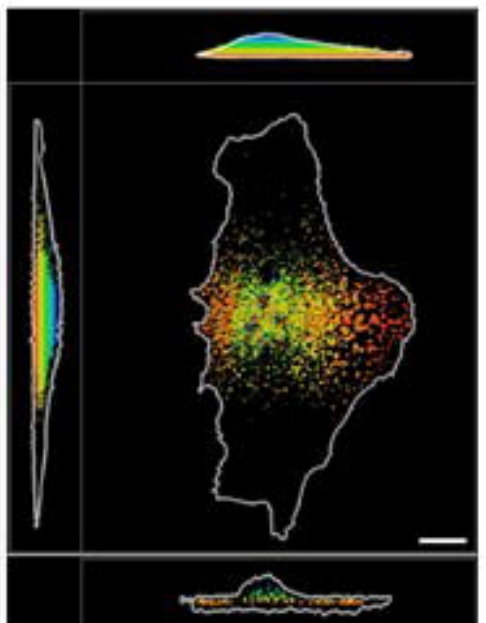

\section{single Bessel beam}
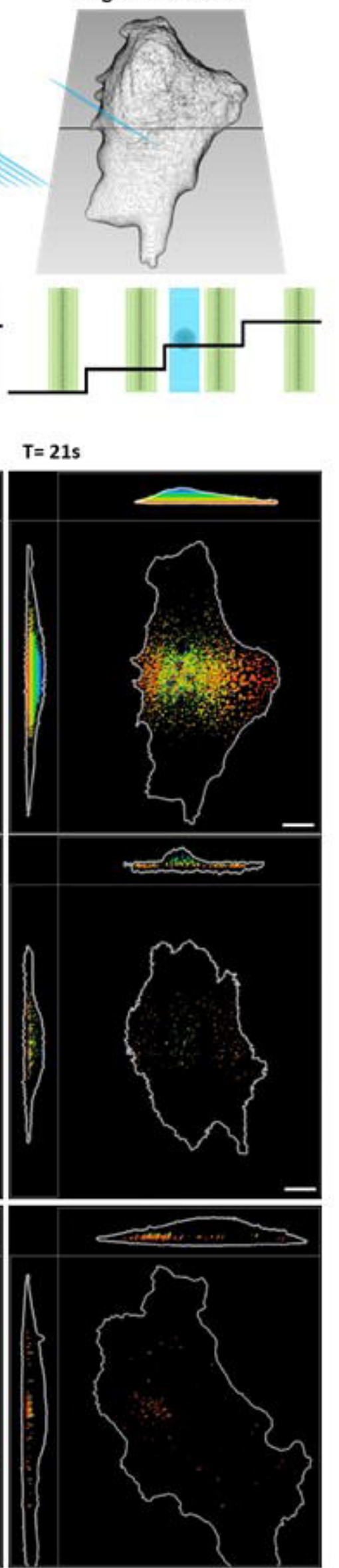

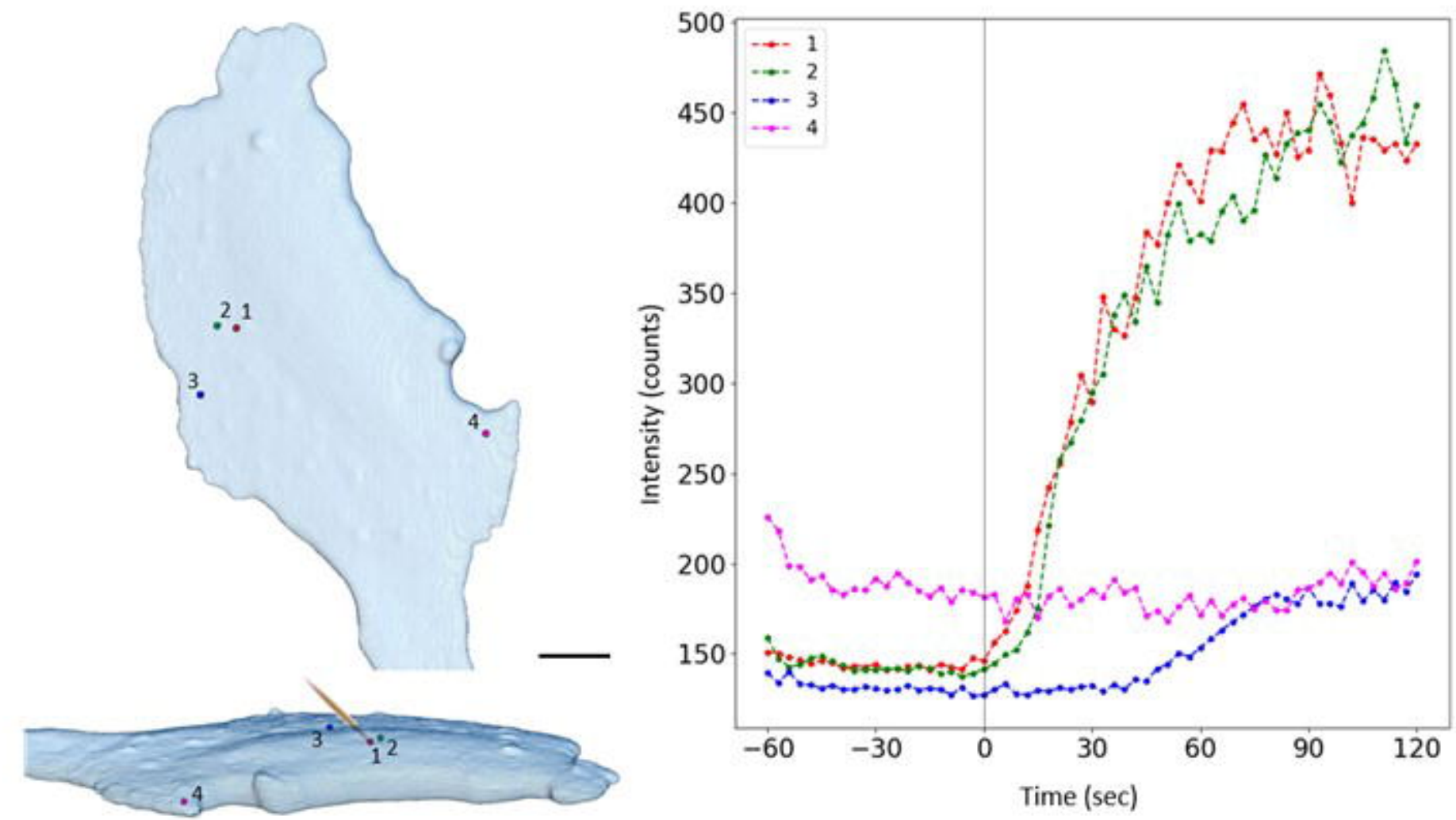

C
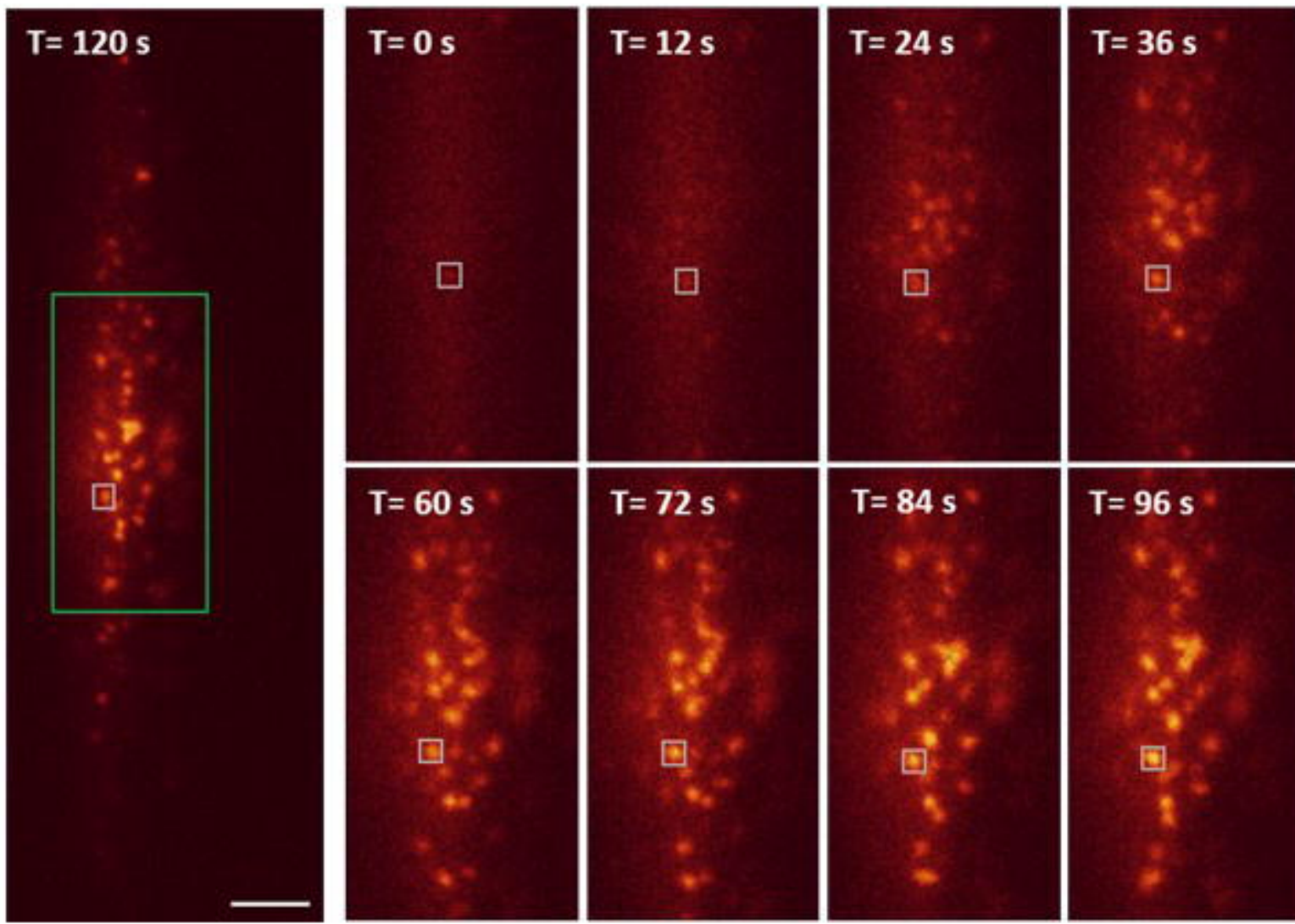

$\mathrm{T}=48 \mathrm{~s}$
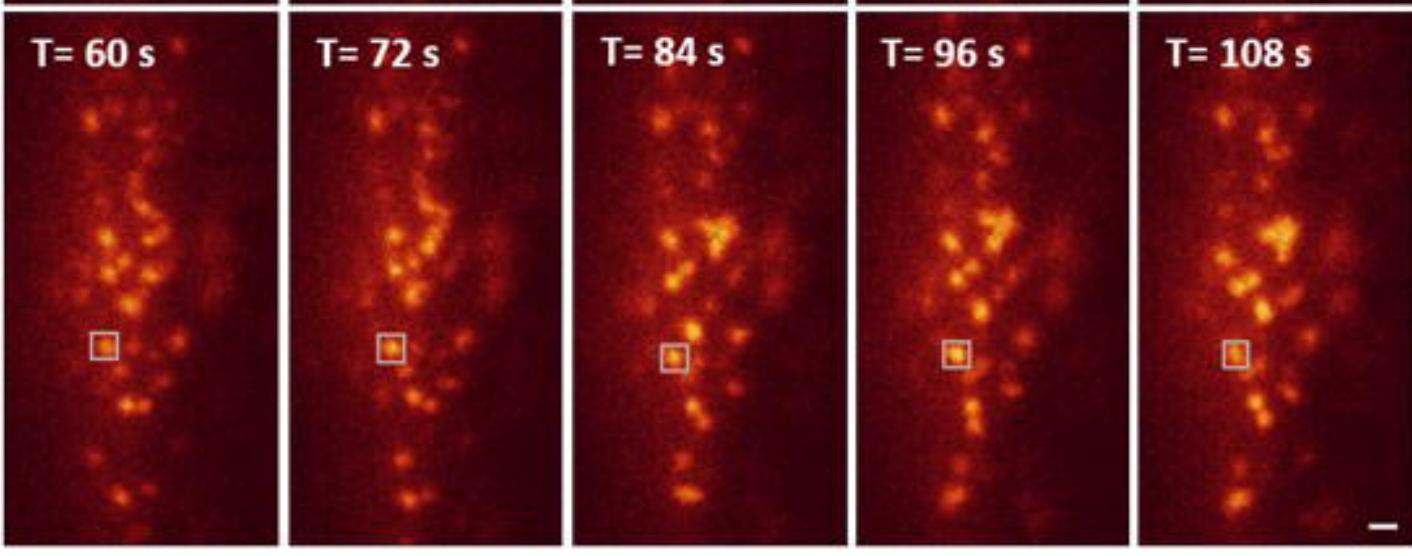
a

خ্য (2)

莚
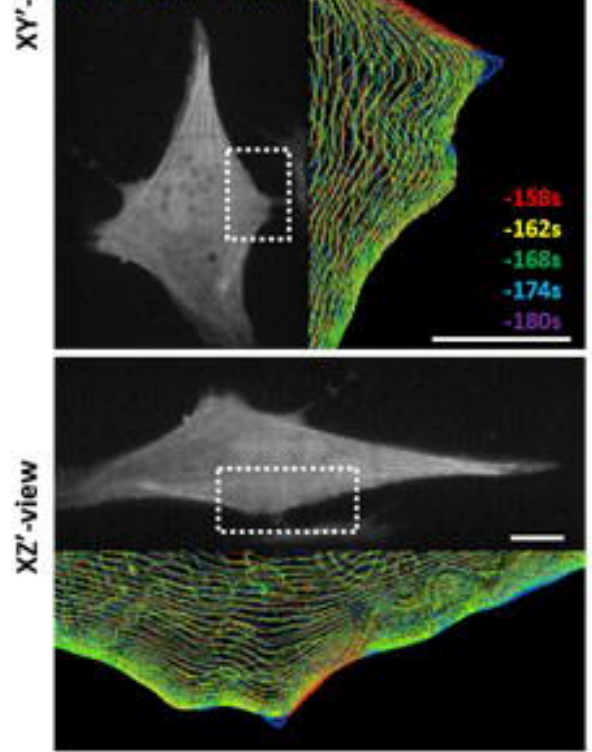

b

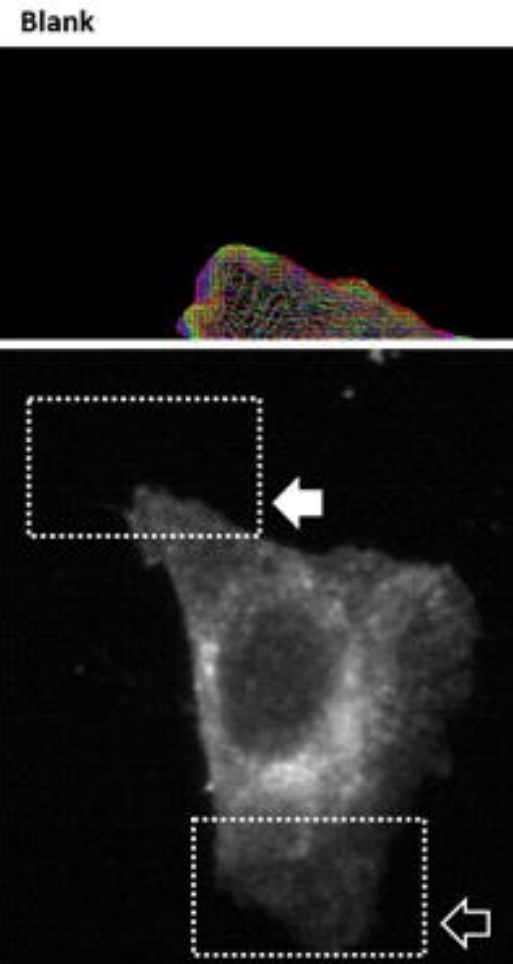

Stimulation (region 1)

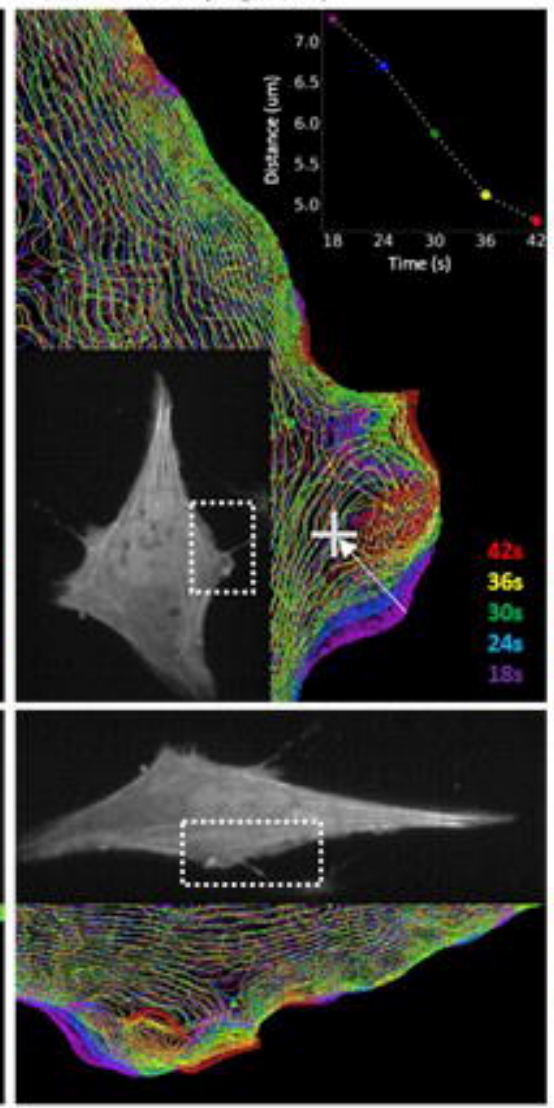

Stimulation (region 1)
Stimulation (region 2)

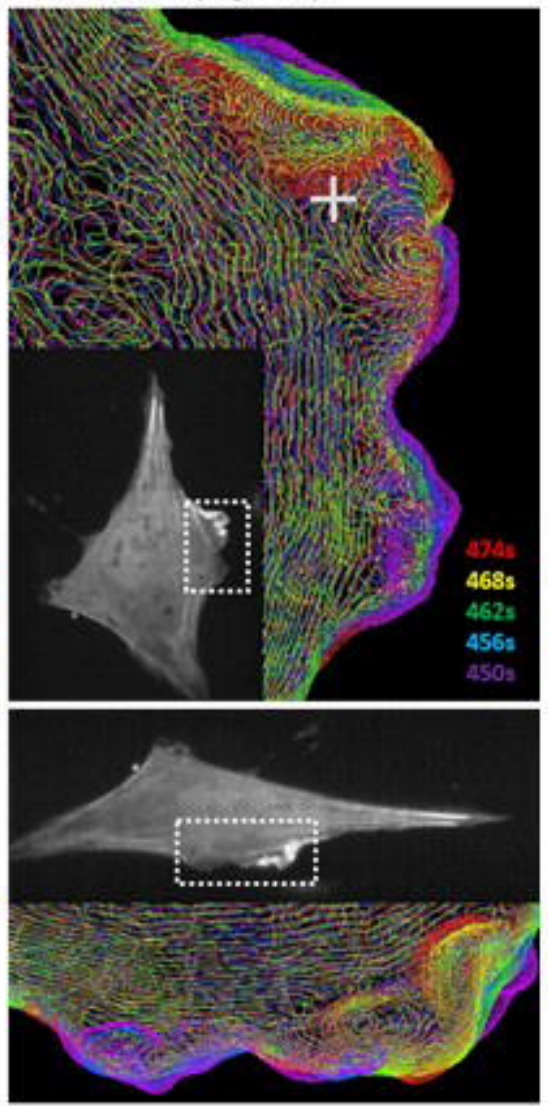

Stimulation (region 2)
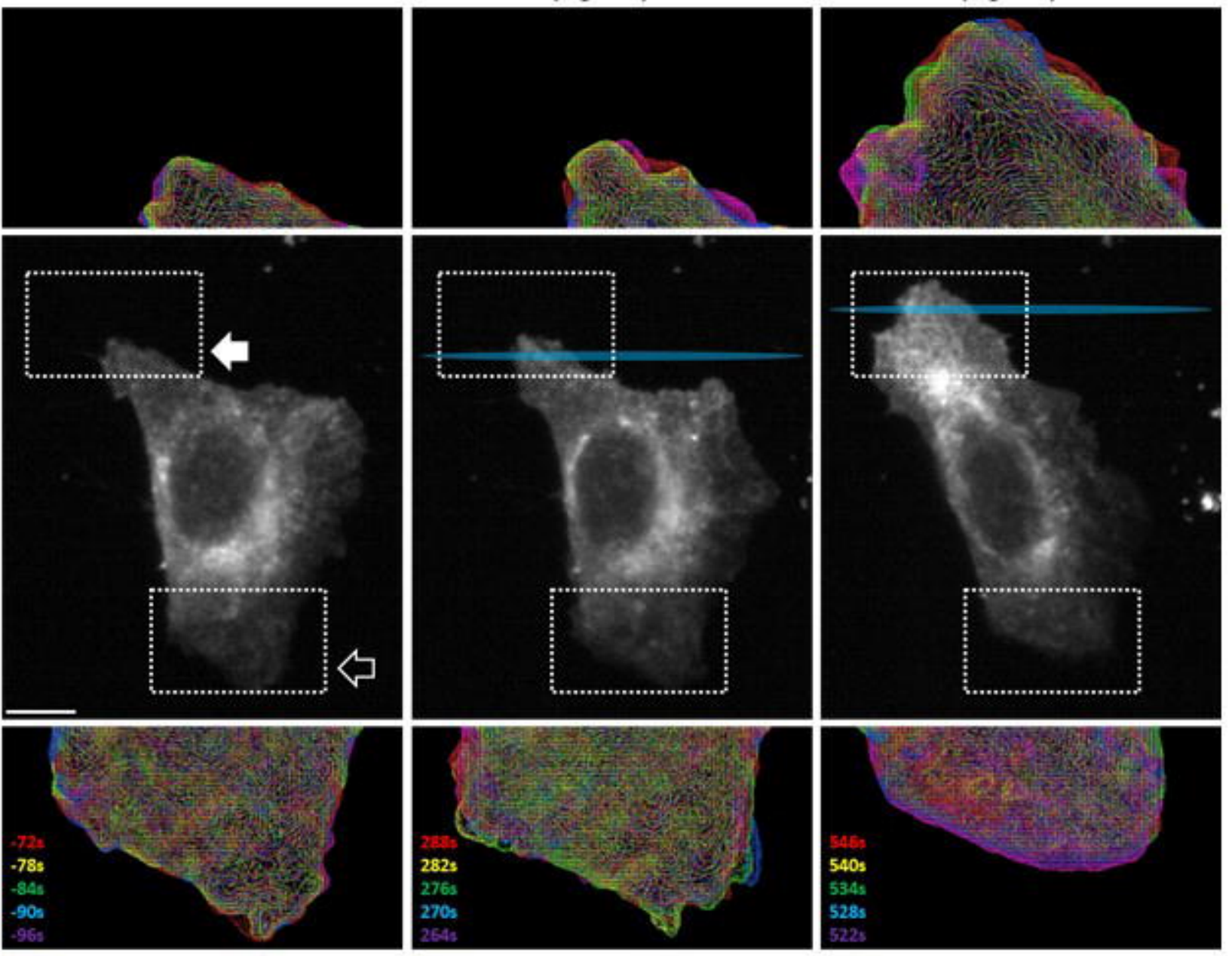


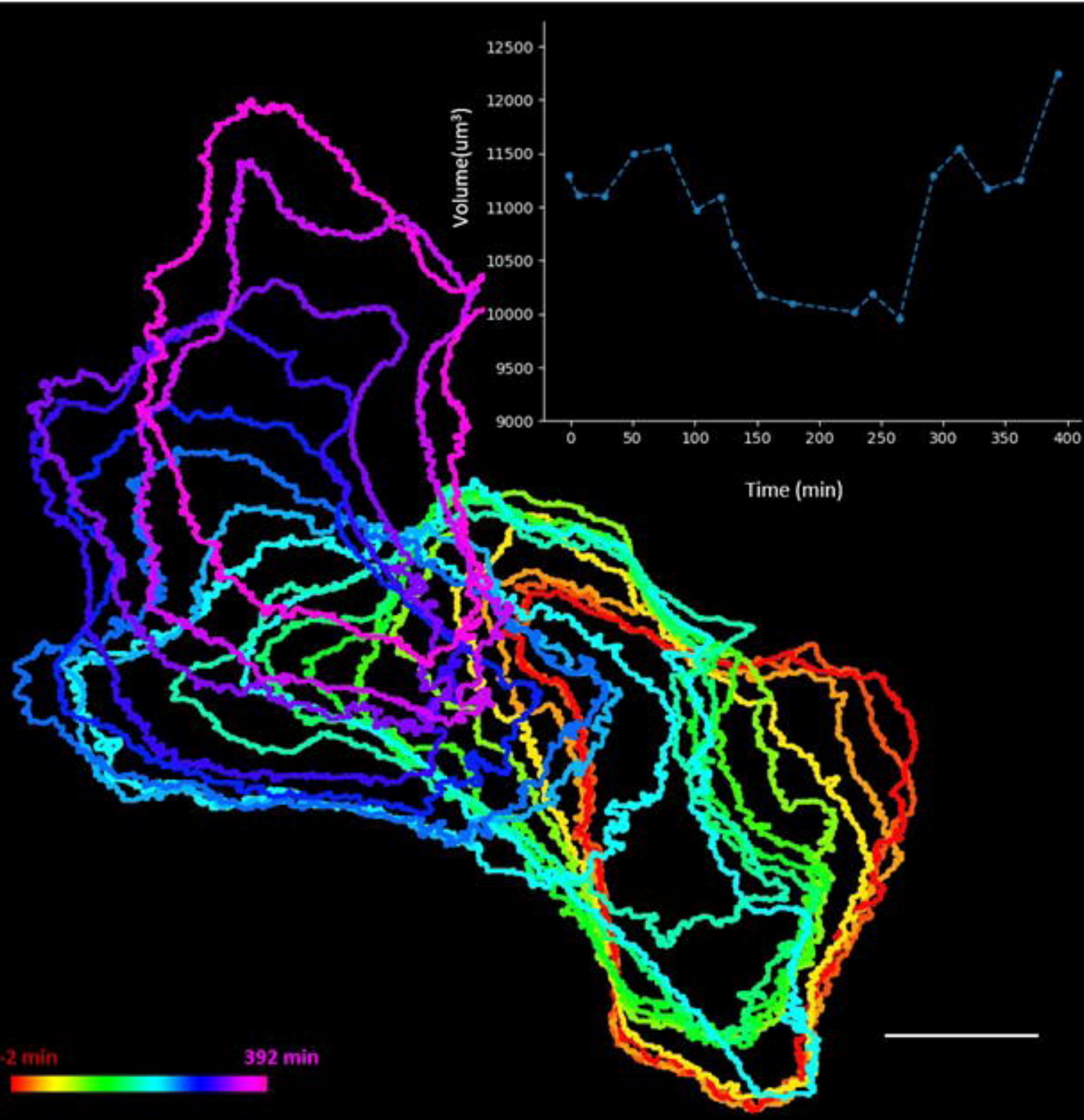

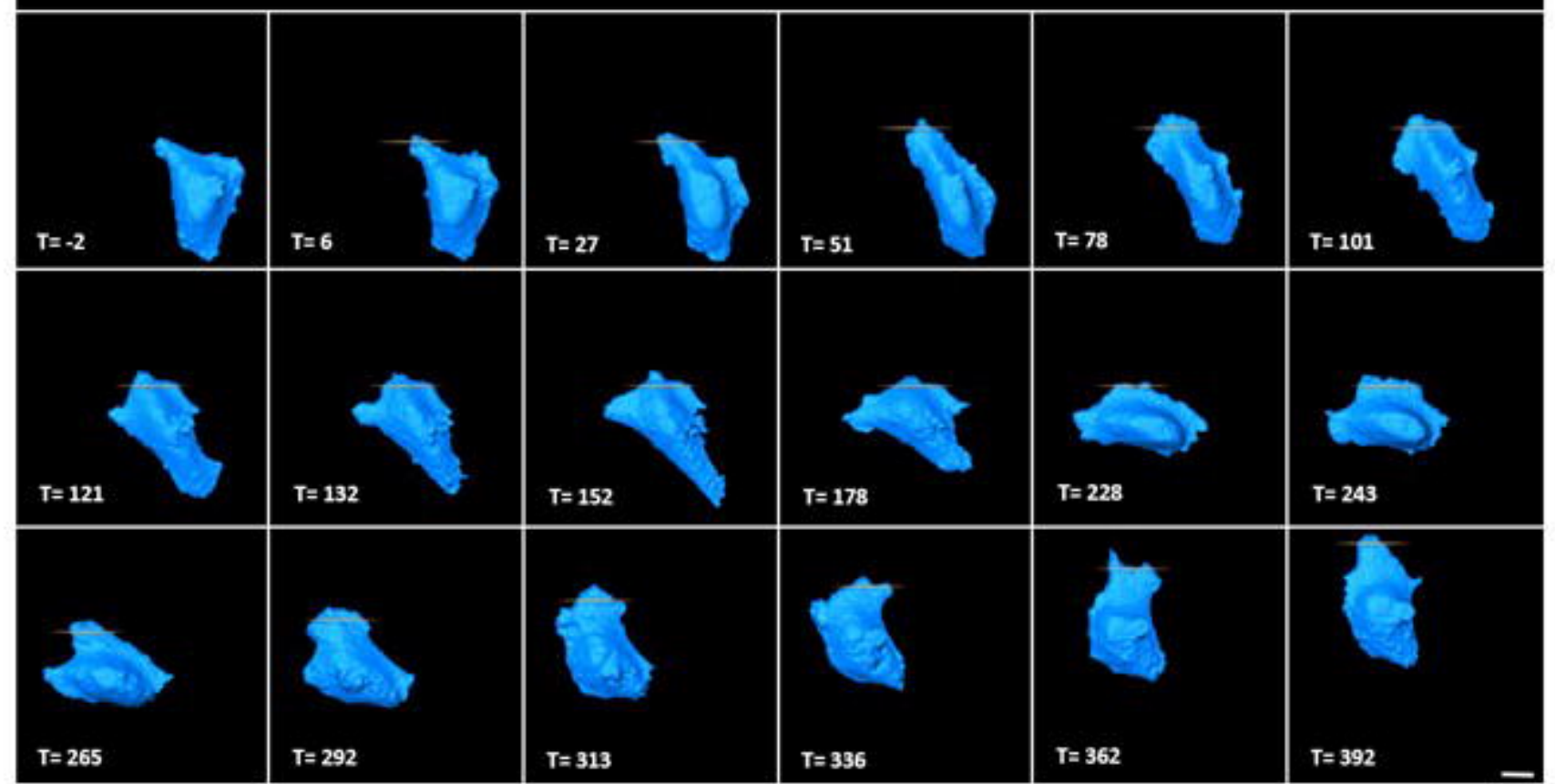

